\title{
Revenue management in a multi-stage make-to-order production system
}

\author{
Hendrik Guhlich ${ }^{a} \&$ Moritz Fleischmann ${ }^{b} \&$ Lars Mönch $^{c} \&$ Raik Stolletz ${ }^{d}$ \\ ${ }^{a}$ University of Mannheim, Business School \\ guhlich@bwl.uni-mannheim.de \\ ${ }^{b}$ University of Mannheim, Business School \\ mfleischmann@bwl.uni-mannheim.de \\ ${ }^{c}$ University of Hagen, Department of Mathematics and Computer Science \\ Lars.Moench@Fernuni-Hagen.de \\ ${ }^{d}$ University of Mannheim, Business School \\ stolletz@bwl.uni-mannheim.de
}

October 14, 2015

\begin{abstract}
In the present paper, we consider the demand management decisions of a manufacturer facing stochastic demand. While in the previous literature, either the order release decisions are fixed upon arrival or a single-stage production environment is assumed, we make online order acceptance and order release decisions in a multi-stage production system. After describing the problem formally as a stochastic dynamic program, we develop a bid-pricebased revenue management approach in which the order acceptance and order release decisions are made based on previously computed bid prices and feasibility according to a linear program describing the multi-stage production system. A numerical study shows the good performance of the approach compared to an ex-post optimal solution in various supply and demand settings as well as the benefits relative to existing models, which work under simplifying assumptions.
\end{abstract}




\section{Introduction}

Revenue management has emerged mainly in the service industry, and most of the literature on the subject also involves this industry; see Talluri and Van Ryzin (2004) for an overview. Nevertheless, revenue management ideas have also been applied in make-to-stock (MTS) production systems (Quante et al., 2009; Meyr, 2009), make-to-order (MTO) production systems (e.g., Easton and Moodie, 1999; Barut and Sridharan, 2005; Kuhn and Defregger, 2005; Spengler et al., 2007; Watanapa and Techanitisawad, 2005; Spengler et al., 2008; Modarres and Sharifyazdi, 2009; Hintsches et al., 2010; Li et al., 2012; Volling et al., 2012; Chevalier et al., 2015), and assemble-to-order (ATO) production systems (Harris and Pinder, 1995; Gao et al., 2012; Guhlich et al., 2015).

In the present paper, we consider an MTO manufacturer using a multi-stage production system. Short-term capacity adjustments are impossible during the considered planning horizon, and the available capacities are tightly planned. Demand is uncertain, fluctuating, and exceeds available capacities during peak phases. Therefore, the scarce resources that are available must be allocated to incoming demand; that is, order acceptance and order release decisions must be made. These decisions are of crucial importance if orders are heterogeneous, i.e., when they differ in capacity requirements and contribution margins.

This is the case, e.g., in the steel industry (Hintsches et al., 2010), where different products are produced to order in a multi-stage no-wait flow shop, and capacity expansion is very costly. Orders differ in their capacity consumption and contribution margins. It is possible to reject orders from customers without long-term contracts because each of these customers has a relatively low overall economic impact.

Another possible field of application is in semiconductor backend facilities in which chips are customized in a multi-stage production system, based on customer-specific requirements (Brown et al., 2000). In this production environment, capacities are also fixed and tightly planned due to high capital investment costs. Again, demand is fluctuating and uncertain.

While many revenue management approaches only consider order acceptance decisions (e.g., Talluri and Van Ryzin, 1999; Spengler et al., 2007; Hintsches et al., 2010), there is typically more than one possible release date for an incoming order in the described production environments. Keeping more flexibility in the production schedule allows the company to accept and produce more orders.

In the present paper, we consider the demand management decisions of an MTO manufacturer using a multi-stage production system under stochastic demand. Incoming orders are accepted (or rejected) immediately upon arrival, while order release decisions are made period- 
ically for the pool of accepted orders. The goal is to maximize the marginal profit generated by the accepted orders, while taking into account holding and backlog costs. To the best of our knowledge, this problem has not been previously addressed in the literature.

The main contributions of the present paper are as follows:

- We introduce a novel revenue management problem, considering online order acceptance and online order release planning in a multi-stage production system, and we model it as a stochastic dynamic program (SDP).

- We develop a heuristic revenue management approach based on bid prices to solve instances of realistic size.

- A numerical study shows the good performance of our approach in different production environments in comparison to an ex-post optimal solution. The study suggests that aggregating the considered multi-stage production systems into single-stage systems leads to unsatisfactory results. In addition, making online order release decisions can lead to significantly greater profits.

The paper is organized as follows. In the next section, an overview of the related literature is given. The problem is formulated in Section 3. In Section 4, we model the problem as an SDP. We develop a bid-price-based revenue management approach in Section 5. A numerical study evaluates the performance of the presented heuristic in Section 6. Section 7 concludes the paper.

\section{Literature review}

In this section, we give a brief overview of bid-price approaches in the revenue management literature. We consider applications in the service and manufacturing industries. In particular, we concentrate on the supported decisions and the production systems considered.

Network revenue management is concerned with managing multiple resources, such as different flight legs (see e.g., Talluri and Van Ryzin, 1998). There is no flexibility to assign customers to different resources (flight legs) after the purchase. Therefore, it is impossible to perform online scheduling in the previous literature, as is done in the present paper.

Revenue management of flexible products (Gallego and Phillips, 2004; Gallego et al., 2004; Petrick et al., 2010, 2012; Gönsch et al., 2014) allows the company to delay the assignment of customers to specific products (e.g., a specific flight). These decisions are made only after all requests for the flexible products have arrived, which enables the company to use capacities more 
efficiently. In contrast, in the problem considered in the present paper, service provision and the arrival of orders cannot be separated because these events overlap. Due to this different time structure, these approaches are not applicable in an online order acceptance and order release planning model. An extensive discussion of this topic can be found in Guhlich et al. (2015).

In revenue management in MTO manufacturing, bid-price approaches have been proposed in the context of steel production. Spengler et al. (2007) consider a multi-stage production model to compute bid prices via a multi-dimensional knapsack formulation. However, only one production start date is available for each arriving order. Therefore, no scheduling decisions are made. In addition, orders released in different planning periods do not share any common resources, which is a major difference to the model used in the present paper. Spengler et al. (2008) propose an extension to this approach that allows for shifting demand between different planning periods. However, the scheduling decisions must be made immediately upon arrival of the order, whereas the online scheduling approach in the present paper can change the release date of an order until its production is actually started. Hintsches et al. (2010) consider long planning periods so that a planning period is long enough that an order finishes production within the same planning period in which it was started. Again, there is only one possible production start date per order.

Guhlich et al. (2015) develop a bid-price-based revenue management approach for ATO manufacturing. In their setting, production capacity and intermediate materials are scarce resources. In addition to order acceptance, they quote a firm due date on the arrival of each order and perform online scheduling. However, in contrast to the present paper, they consider a single-stage production model.

To sum up, no approach from the existing literature is capable of making online order acceptance and online order release decisions in a multi-stage production environment. Either the order release decisions are fixed upon order arrival, or a single-stage production environment is assumed.

\section{Problem formulation}

In this section, we state the basic assumptions of the considered planning problem. We describe the production system, the production capacity, the orders, the decisions, and the cost structure. Table 1 summarizes the notation.

The planning horizon is divided into planning periods, $t=1, \ldots, T$. Each planning period $t$ is further divided into micro-periods $(t, s)(s=1, \ldots, S)$ such that in each of these micro-periods, one order arrives at most. Let $d=1, \ldots, D$ denote the incoming orders. We make the following 


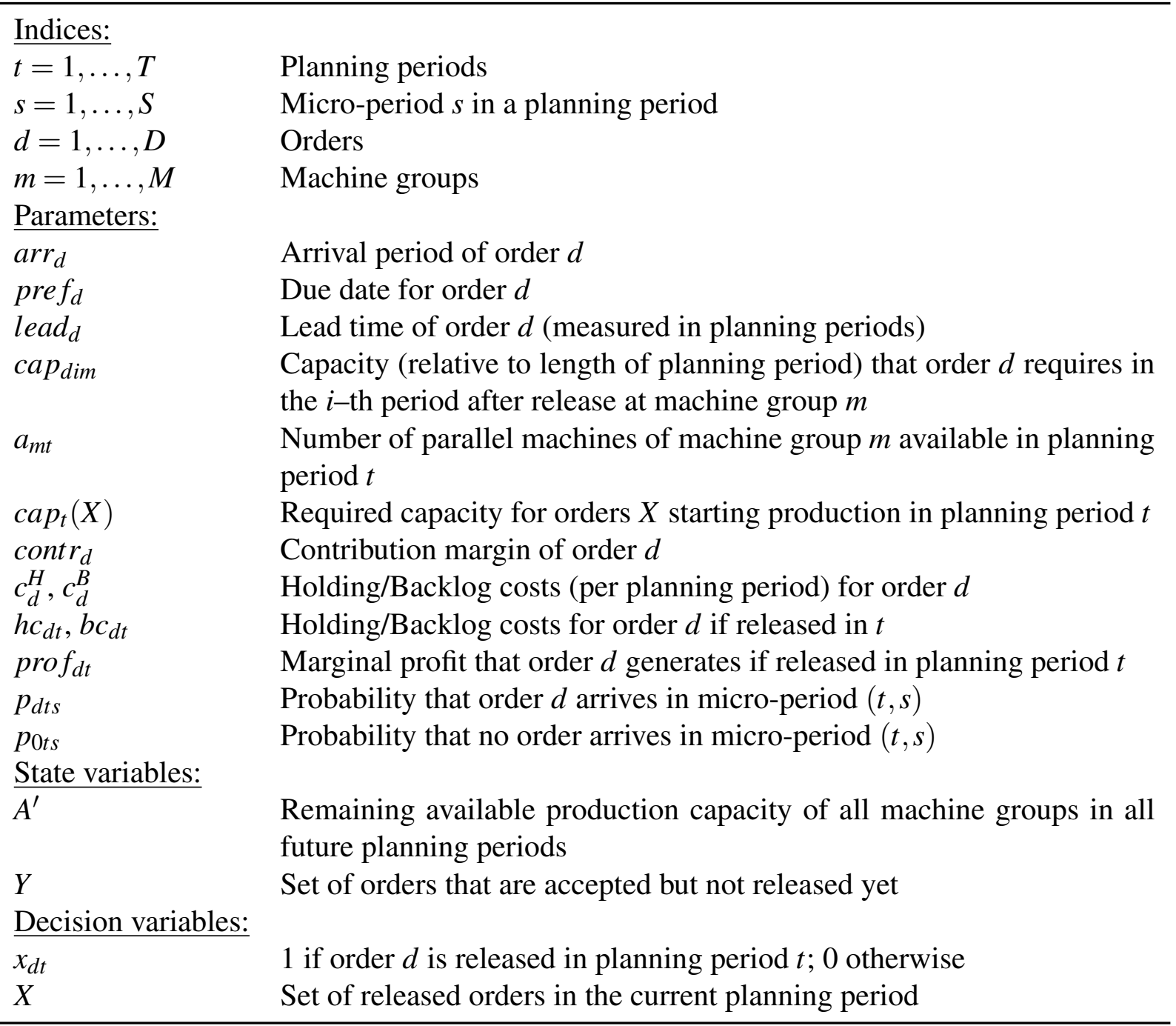

Table 1: Notation 


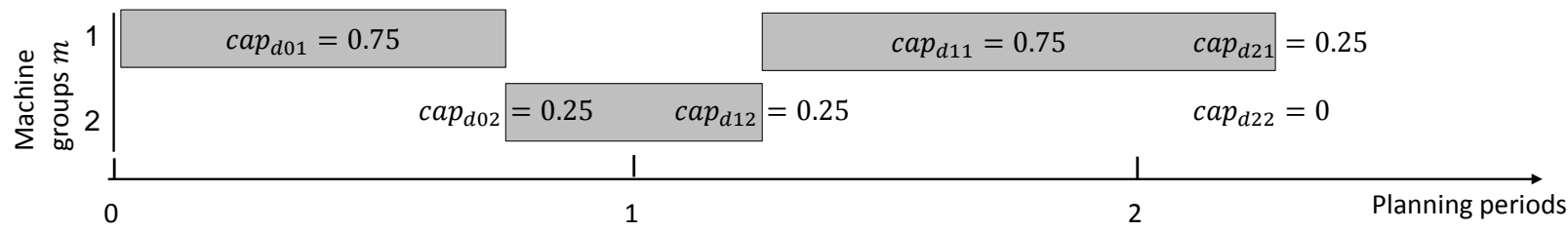

Figure 1: Example of a Gantt chart for an order $d$ with lead time $\operatorname{lead}_{d}=3$

assumptions:

Assumption 3.1 (Production system). We consider a no-wait, flexible flow shop production system consisting of machine groups $m=1, \ldots, M$, with $a_{m t}$ identical parallel machines available for machine group $m$ in planning period $t$.

In a no-wait production system, orders are processed without waiting after their release. This requirement stems either from technical factors, as in the steel industry (Wismer, 1972; Tang et al., 2001), or from the fact that no intermediate storage is available. Hall and Sriskandarajah (1996) give an overview of industries using this type of production system.

Assumption 3.2 (Production capacity). Each order can only be processed by one machine at a time. The production of an order d requires cap dim time units of capacity (relative to the length of a planning period, i.e., $0 \leq$ cap $_{\text {dim }} \leq 1$ ) in the $i$-th planning period after starting production at machine group $m$. The lead time, lead , is measured by the number of required planning periods. See Figure 1 for an example. Setup times are not considered, and processing times are deterministic.

Let $A^{\prime}$ denote the matrix of the remaining free production capacities in the remaining planning periods at all machine groups. For a set of orders $X$ released in planning period $t$, cap $_{t}(X)$ is defined as the matrix of required production capacities (in all future planning periods at all machine groups). Its $(i, m)$-th entry describes the total capacity consumption at machine group $m$ in period $t+i$ and is defined as $\sum_{d \in X}$ cap dim.

Assumption 3.3 (Orders). Orders arrive according to a known probability distribution. The probability that order $d$ arrives in micro-period $(t, s)$ is given by $p_{d t s}$. The probability that no order arrives in micro-period $(t, s)$ is given by $p_{0 t s}$. Each order $d$ has the following characteristics:

- Planning period that contains the micro-period in which the order arrived $\left(\operatorname{arr}_{d}\right)$

- Due date $\left(\right.$ pref $\left._{d}\right)$ 
- Capacity requirement at machine group $m$ in the $i-$ th period after release (cap dim)

- Lead time $\left(\right.$ lead $\left._{d}\right)$

- Holding and backlog cost rates $\left(c_{d}^{H}, c_{d}^{B}\right)$

- Contribution margin (contr ${ }_{d}$ ).

Assumption 3.4 (Decisions). The company makes the following decisions:

1. Order acceptance: In each micro-period, the incoming order is immediately accepted or rejected.

2. Order release: At the beginning of each planning period, the set of orders to be released, $X \subseteq Y$, is selected.

Here, $Y$ denotes the set of orders that are currently accepted but not yet released. Orders can only be fully accepted and fully released to the shop floor. Orders can be released at the earliest in the first planning period after their arrival.

Assumption 3.5 (Cost structure). If an accepted order is not fulfilled by the due date, backlog costs are incurred for each planning period beyond this date. Holding costs are incurred for orders finishing before the due date.

If production for order $d$ starts in planning period $t$, holding costs $h c_{d t}$ and backlog costs $b c_{d t}$ for this order are given by:

$$
\begin{aligned}
h c_{d t} & :=\max \left\{0, \text { pref }_{d}-\left(t+\text { lead }_{d}\right)\right\} \cdot c_{d}^{H} \\
b c_{d t} & :=\max \left\{0,\left(t+\text { lead }_{d}\right)-\text { pref }_{d}\right\} \cdot c_{d}^{B} .
\end{aligned}
$$

If the production of an order $d$ starts in planning period $t$, the following marginal profit is earned:

$$
\operatorname{prof}_{d t}:=\operatorname{contr}_{d}-h c_{d t}-b c_{d t} .
$$

\section{Stochastic dynamic program}

In this section, we formalize the aforementioned problem by formulating it as an SDP. Specifically, we describe the state space, the decisions and transitions, the Bellman equation, and the feasibility check. Table 2 summarizes the additional notation. 


\begin{tabular}{ll}
$Z$ & $\begin{array}{l}\text { Set of all feasible states } \\
\left(A^{\prime}, Y\right)\end{array}$ \\
$\begin{array}{l}\text { State of the system given by remaining available capacity } A^{\prime} \text { and orders } \\
Y \text { that have been accepted but not yet released } \\
V_{t s}\left(A^{\prime}, Y\right)\end{array}$ \\
$\begin{array}{l}\text { Maximum expected marginal profit-to-go in state }\left(A^{\prime}, Y\right) \text { in planning } \\
\text { period } t \text { if } s=0 \text { and micro-period }(t, s) \text { otherwise }\end{array}$ \\
\hline
\end{tabular}

Table 2: Additional notation for the SDP

\section{State space}

The state of the system is given by $\left(A^{\prime}, Y\right)$, the remaining available production capacity $A^{\prime}$ at each machine group in the upcoming planning periods and the set of orders $Y$ that are accepted but not yet released.

Let $Z$ define the set of all feasible states. A state is feasible if all accepted orders can be produced within the planning horizon. This can be checked using linear constraints, as defined at the end of this section.

\section{Decisions and transitions}

In each micro-period $(t, s)$, the decision of whether to accept the arriving order is made. If the order is rejected (or no order arrives), the state remains unchanged. Otherwise, the accepted order $d$ is added to the set of orders that are accepted but not yet released, denoted by $Y \cup d$, and the contribution margin of the order is earned.

At the beginning of each planning period $t$, the set of orders released $X \subseteq Y$ is determined. These orders are removed from $Y$ (denoted by $Y \backslash X$ ), and the available capacities are updated (denoted by $A^{\prime}-\operatorname{cap}_{t}(X)$ ). Additionally, holding and backlog costs are determined for newly released orders.

\section{Profits}

Let $V_{t s}\left(A^{\prime}, Y\right)$ be the maximum expected marginal profit-to-go in state $\left(A^{\prime}, Y\right)$ at the beginning of planning period $t$ if $s=0$ and in micro-period $(t, s)$ for $s=1, \ldots, S$, otherwise. Additionally, we define $V_{t, S+1}\left(A^{\prime}, Y\right):=V_{t+1,0}\left(A^{\prime}, Y\right)$. Then, $V_{t s}$ can be computed recursively via the Bellman 
equation for $\left(A^{\prime}, Y\right) \in Z$ as follows:

$$
V_{t s}\left(A^{\prime}, Y\right)= \begin{cases}\sum_{d} p_{d t s} \cdot \max \{\overbrace{V_{t, s+1}\left(A^{\prime}, Y \cup d\right)+\text { contr }_{d},}^{\text {accept d }} \overbrace{V_{t, s+1}\left(A^{\prime}, Y\right)}^{\text {reject d }}\}+\overbrace{p_{0 t s} \cdot V_{t, s+1}\left(A^{\prime}, Y\right)}^{\text {no order }} & \text { if } 1 \leq s \leq S \\ \underbrace{\max _{X \subseteq Y}\left\{V_{t, s+1}\left(A^{\prime}-\operatorname{cap}_{t}(X), Y \backslash X\right)-\sum_{d \in X}\left(h c_{d t}+b c_{d t}\right)\right.}_{\text {order release decision }}\} & \text { if } s=0\end{cases}
$$

with boundary conditions $V_{T, S+1}\left(A^{\prime}, Y\right)=0$ for $\left(A^{\prime}, Y\right) \in Z$ and $V_{t s}\left(A^{\prime}, Y\right)=-\infty$ for $\left(A^{\prime}, Y\right) \notin Z$.

We can use this SDP (4) to compare the considered problem with other models from the literature. The main difference from models in the literature on MTO-RM (Spengler et al., 2007; Hintsches et al., 2010) and FP-RM (Gallego et al., 2004; Petrick et al., 2010, 2012) is that in the SDP (4), there are two different time levels at which decisions are made. Order acceptance decisions are made immediately after order arrival, while order release decisions are only made at the beginning of each planning period. Compared to Guhlich et al. (2015), in the present paper, multiple machine groups are considered, while Guhlich et al. (2015) assume a single-stage production system. However, in the present paper, no intermediate materials are considered, and no due dates are quoted upon order arrival. Additionally, orders can only be fully released.

\section{Feasibility check}

To check the feasibility of a state, that is to check whether $\left(A^{\prime}, Y\right) \in Z$ in planning period $t^{f i x}$, we use a set of linear constraints. To this end, we define decision variables $x_{d t}$ for each order $d$ and planning period $t$ with $\max \left(a r r_{d}, t^{f i x}\right)+1 \leq t \leq T-$ lead $_{d}$, which model the decision if order $d$ is released in planning period $t$. The following constraints must be satisfied: ${ }^{1}$

$$
\begin{array}{rlrl}
\sum_{d \in Y} \sum_{i=0}^{\text {lead }_{d}-1} \operatorname{cap}_{\text {dim }} \cdot x_{d, t-i} & \leq a_{m t}^{\prime} & & \text { for } m=1, \ldots, M ; t=t^{f i x}+1, \ldots, T \\
\sum_{t=\text { arr }_{d}+1}^{T-\text { lead }_{d}} x_{d t} & =1 & & \text { for } d \in Y \\
x_{d t} & \in\{0,1\} & \text { for } d \in Y ; t=t^{f i x}+1, \ldots, T .
\end{array}
$$

Constraint (5) describes the capacity consumption. The used capacity, which is composed of

\footnotetext{
${ }^{1}$ We follow the convention that undefined variables are equal to 0 .
} 
the different orders that may require machine group $m$ in different planning periods after their start, must not exceed the available capacity. Constraint (6) guarantees that orders that have been accepted in previous planning periods will be finished within the planning horizon. Constraint (7) ensures that orders can only be fully released.

Constraints (5)-(7) are also the basis for the revenue management approach presented in the next section.

\section{Revenue management approach}

Because of the high dimensionality of the state space, it is computationally intractable to solve the SDP described in the previous section for instances of realistic size (cf. Petrick et al., 2012). Therefore, in this section, we develop a bid-price-based revenue management approach to solve the problem heuristically.

The general approach is as follows:

1. At the beginning of the planning horizon, bid prices are computed using randomized linear programming (RLP) (Talluri and Van Ryzin, 1999). Bid prices are used as a measure for the opportunity costs of consuming resources.

2. Upon arrival, we decide whether to accept an incoming order based on the previously computed bid prices and the feasibility according to the modeled production system. An order is accepted if there is a feasible release date for which the incremental profit exceeds the estimated opportunity costs of the required resources. The feasibility check is performed using constraints (5) - (7).

3. At the beginning of each planning period, order release decisions are made for the current planning period, considering all orders that are accepted but not yet released. To this end, a suitable objective function (using bid prices) is defined for constraints (5) - (7).

In the following, we describe in detail how to derive bid prices and how to use them to make order acceptance and order release decisions.

\subsection{Bid price computation}

To derive bid prices, the problem is modeled as a profit-maximizing non-integer linear program where future demand is assumed to be known. As known from the literature (Simpson, 1989; Williamson, 1992; Talluri and Van Ryzin, 2004), the derived primal optimal decisions can be 
discarded, and the shadow prices of the variables corresponding to the resource constraints are used as bid prices. They are a measure for the opportunity costs of using the corresponding resources. In the RLP, several demand scenarios $\mathcal{D}_{j}$ are generated, and the mean over the resulting bid prices is taken (Talluri and Van Ryzin, 1999). We update the bid prices iteratively using new demand scenarios until the average over all computed bid prices converges, as described in Guhlich et al. (2015). Hence, the number of scenarios is not fixed beforehand.

For scenario $j$, the objective function to be maximized is defined as follows:

$$
\sum_{d \in Y \cup \mathcal{D}_{j}} \sum_{t=\operatorname{arr}_{d}+1}^{T-\text { lead }_{d}} \operatorname{prof}_{d t} \cdot x_{d t}
$$

In addition to Constraints (5) and (6), for each scenario $j$, the following inequalities must hold to guarantee that not more is produced than is demanded:

$$
\sum_{t=\text { arr }_{d}+1}^{T-\text { lead }_{d}} x_{d t} \leq 1 \quad \text { for } d \in \mathcal{D}_{j}
$$

We no longer force the decision variables $x_{d t}$ to be integer because doing so would hinder shadow prices from being easily derived from the linear program. Relaxing the integrality constraints is also common practice in the classical revenue management approaches in the airline industry (cf. Talluri and Van Ryzin (2004)). Constraints (7) are therefore relaxed to $x_{d t} \geq 0$ for all orders $d \in Y \cup \mathcal{D}_{j}$, which allows for releasing fractions of an order in different planning periods.

The dual variables corresponding to the capacity constraints (5) in an optimal solution are then used as scenario bid prices $b p_{j}(m, t)$ for using machine group $m$ in planning period $t$ in scenario $j$. Production capacity that is unused in the current planning period is lost so that bid prices concerning these resources can be set to zero. The overall bid prices $b p(m, t)$ are then determined as the average over all scenario bid prices $b p_{j}(m, t)$.

\subsection{Order acceptance decision}

Following the approaches of Petrick et al. (2012) and Guhlich et al. (2015), we define the opportunity costs of accepting order $d$ and releasing it in planning period $t$ as

$$
\operatorname{opp}^{\text {res }}(d, t):=\sum_{m=1}^{M} \sum_{i=0}^{\text {lead }_{d}-1} \operatorname{cap}_{\text {dim }} \cdot b p(m, t+i) .
$$


An order $d$ is accepted if there exists a planning period $t$ in which it is feasible to release $d$ and

$$
\operatorname{prof}_{d t} \geq o p p^{r e s}(d, t) .
$$

At this point in time, the order release date is still subject to change. Note that this approach underestimates the resulting backlog costs if accepting order $d$ increases the backlog costs of one of the already accepted orders $Y$.

\subsection{Order release decision}

At the beginning of each planning period $t$, we must decide which of the accepted orders to release onto the shop floor. To take into account future arriving demand, we follow the approach of Guhlich et al. (2015) and maximize

$$
\sum_{d \in Y} \sum_{t=f^{f i x}}^{T-\text { lead }_{d}}\left(\operatorname{prof}_{d t}-o p p^{r e s}(d, t)\right) \cdot x_{d t}
$$

with respect to constraints (5) - (7) to obtain a preliminary schedule. Only the order release decisions in the current planning period $t^{\text {fix }}$ are implemented, while order release decisions for future periods are discarded. At the end of each planning period, the remaining available production capacity $A^{\prime}$ is updated.

\section{Numerical study}

In this section, we numerically investigate the performance of the revenue management approach presented in the previous section. First, we describe the experimental design. Next, we show that the proposed revenue management approach works well. Finally, the impact of aggregating the production systems to single-stage systems and the impact of making order release decisions online is examined.

\subsection{Experimental design}

In the following, we describe the parameter settings, production systems, and algorithms used in the experiments. 


\section{Parameter settings}

In all simulations, we consider a planning horizon of $T=40$ planning periods. Three different order types are available that all require the same resources but differ in their contribution margins. The due date depends on the order type. Order types that are more valuable arrive with an earlier due date because the willingness to pay is higher for customers with urgent orders. Therefore, high value orders can be delayed by only one planning period before incurring backlog costs. The due date for medium value orders is two planning periods after the earliest possible finish date, and low value orders can be delayed by four planning periods without incurring backlog costs. We obtain:

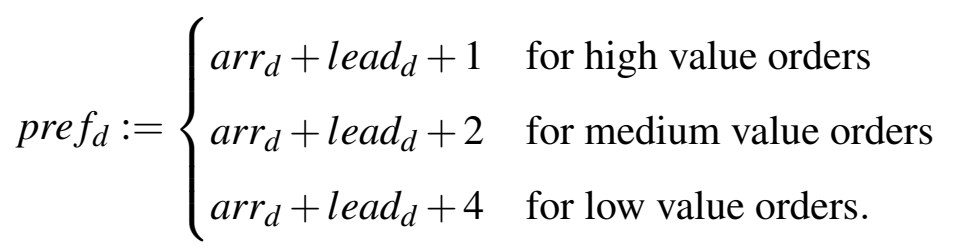

Holding and backlog cost rates are 3\% and 5\% of the contribution margin, respectively.

The performance of the algorithm depends on the considered demand scenarios. In particular, we expect that the profit heterogeneity of the orders, the scarcity level of capacity, defined as the ratio between demand and available capacity, and the coefficient of variation of the probability distribution for the incoming demand have a strong influence. Therefore, we vary these parameters as summarized in Table 3.

The profit heterogeneity in a demand scenario depends on the contribution margins for the $\mathrm{high} /$ medium/low-value orders. In the scenarios with low profit heterogeneity, the contribution margin of a high-value order is twice the contribution margin of a low-value order, while in a scenario with high profit heterogeneity, they differ by a factor of 5 .

The number of arriving orders in each planning period is drawn from a negative binomial distribution $N B(\mu, C V)$ with mean $\mu$ and coefficient of variation $C V$. This distribution is commonly used in the literature (Ehrenberg, 1959; Agrawal and Smith, 1996; Quante et al., 2009; Guhlich et al., 2015). The mean number of arriving orders is varied, resulting in different levels of scarcity of the capacity. We use a $C V$ of 0.5 and 0.75 to represent different levels of demand uncertainty.

For each factor combination from Table 3, 30 independent demand scenarios are generated. This leads to 540 instances for a given production system.

Note that we use a different order arrival structure than in the SDP (4) because in practice, the number of arriving orders within a given planning period is easier to forecast, while the arrival 


\begin{tabular}{lcc}
\hline Factor & Level & Count \\
\hline Contribution margin contr $_{d}$ & $\{(200 / 150 / 100)$, & 3 \\
dependent on order type & $(300 / 200 / 100)$ & \\
& $(500 / 300 / 100)\}$ & \\
Scarcity of capacity & $\{100 \%, 110 \%, 120 \%\}$ & 3 \\
Coefficient of variation $C V$ & $\{0.5,0.75\}$ & 2 \\
\hline
\end{tabular}

Table 3: Parameters used in the numerical study

\begin{tabular}{|c|c|c|c|c|}
\hline & & $M$ & Capacity & Order in which machines are visited \\
\hline 5Stage & & 5 & $a_{m t}=50 \forall m$ & $1,2,3,4,5$ \\
\hline 10Stage & & 10 & $a_{m t}=25 \forall m$ & $1,2,3,4,5,6,7,8,9,10$ \\
\hline Bottle & & 5 & $\begin{array}{c}a_{m t}=50 \forall m \neq 3 \\
a_{3 t}=40\end{array}$ & $1,2,3,4,5$ \\
\hline Reent & & 4 & $\begin{array}{c}a_{m t}=50 \forall m \neq 2 \\
a_{2 t}=100\end{array}$ & $1,2,3,2,4$ \\
\hline 2Prod & $\begin{array}{l}\text { Product } 1 \\
\text { Product } 2\end{array}$ & 5 & $\begin{array}{c}a_{m t}=75 \text { for } m=1,2 \\
a_{m t}=50 \text { for } m=3,4,5\end{array}$ & $\begin{array}{l}1,2,2,3,5 \\
1,1,2,4,5\end{array}$ \\
\hline
\end{tabular}

Table 4: Production systems

structure in the SDP is a standard formulation from the literature.

To avoid the effects of an empty system, the production system is initially filled with a set of orders at different stages of production. This set of orders is generated using the set of orders present in a production system (work in process) after some planning periods, where the presented revenue management approach was applied with a scarcity of capacity of $100 \%$ and a coefficient of variation of 0.5 .

\section{Production environments}

To investigate the impact of different characteristics of a production system on the performance of the tested algorithms, we consider multiple types of production systems. Specifically, we consider five basic systems to examine the single characteristics of production systems and one practice-inspired production system that has a more complex structure. For the basic production systems, Table 4 shows the number of machine groups, the available production capacities, and the sequence in which the machine groups are visited, i.e., the routes of the orders. Each machine 
group is always required for an entire planning period. At the same time, a machine group might be used in multiple consecutive planning periods.

We consider a

- balanced 5-stage production system (5Stage),

- a balanced 10-stage production system (10Stage),

- a 5-stage production system with a bottleneck (Bottle),

- a 4-stage production system with re-entrant flows (Reent),

- a 5-stage production system with two different product types (2Prod),

- and a more complex production system (Complex).

The complex production system is motivated by a real-world semiconductor backend facility (Ehm et al., 2011). It consists of $M=23$ machine groups. Two different product types with different routes through the production system are considered. The required capacities and the number of parallel machines for each machine group are given in the appendix in Tables 11, 12, and 13. We use the same machines and processing times as described in Ehm et al. (2011). For simplicity, however, we exclude some additional characteristics of the production system, namely splitting orders, batching, sequence-dependent setup times and random machine down times.

In the production systems with two different product types, three different order types exist for each product type. The order arrival rates are the same for each order type in each considered production system.

\section{Algorithms}

All algorithms are implemented in C++ using the Gurobi 6.02 solver on a $3.20 \mathrm{GHz}$ Intel Core i7 machine with $32 \mathrm{~GB}$ of RAM.

The presented revenue management approach $(M S-R M)$ is implemented as described in Section 5. Bid prices are recomputed at the beginning of planning periods 10, 20, and 30. The convergence of the bid prices is defined as follows: We look at the set of bid prices generated by including the 10 most recent demand scenarios. We add the new bid prices one by one and check whether one of the mean bid prices changes by more than 5 units. If this is not the case, we say that the bid prices have converged. To avoid premature convergence, the minimum number of 
used demand scenarios is set to 10 . The maximum number of considered demand scenarios is 50.

In the numerical experiments, we compare MS-RM with the following benchmark algorithms:

- First-come-first-serve (FCFS): The FCFS heuristic is wide spread and used in production planning and control (PPC) because of its simplicity. Here, to avoid excessive backlogging, orders are accepted as long as capacity is available to produce the arriving order without backlogging independent of the profit margin. Orders are released to the shop floor as soon as capacity is available.

- Revenue management approaches from the literature considering either a simplified production system or not using online order release decisions

- SingleStage: The SingleStage approach is based on the ATO-RM approach presented in Guhlich et al. (2015). This algorithm models only one machine group. To apply this algorithm, the production systems considered must thus be aggregated to a single-stage production system. To this end, the capacity, which is determined by the number of parallel machines, of this new machine group is chosen such that the resulting maximum throughput is equal to the maximum throughput of the real system. As in the ATO-RM approach, an order requires the capacity of the only available machine group in each planning period during processing, the number of parallel machines is equal to the throughput multiplied by the (number of planning periods of) lead time. If multiple product types are available, the throughput is multiplied with the mean processing time of the product types weighted by their arrival rate. The available capacity in each planning period in the considered production systems is presented in Table 5.

- SchedArr: SchedArr uses the same setup as MS-RM, except that the order release date for this order is decided upon order arrival as described by Spengler et al. (2008). An order $d$ is released in the planning period $t$ so that the difference between the marginal profit prof $f_{d t}$ and the opportunity costs $o p p^{r e s}(d, t)$ is maximized when this maximum is non-negative. The order release date is fixed from this point on.

Because it is computationally intractable to compute an optimal policy for problems of realistic size, we use an ex-post optimal solution (PostOpt) that can be derived from solving the Integer Program consisting of constraints (5), (7), and (9) with objective function (8) under the 


\begin{tabular}{cccccc}
\hline 5Stage & 10Stage & Bottle & Reent & 2Prod & Complex \\
\hline 250 & 250 & 200 & 250 & 250 & 248.81 \\
\hline
\end{tabular}

Table 5: Available capacity for SingleStage in the considered production systems

assumption of known future demand. Notably, the results generated with this approach are only an upper bound of the maximum attainable profit because this algorithm has complete demand information, while the other approaches learn the incoming demand only when the orders arrive.

Except for the Complex production system, all algorithms operate with a planning period length such that no time discretization defects occur. As known from the literature (Hackman and Leachman, 1989; Stadtler, 2008), an exact model of a production system must consider all points in time at which a processing step may finish. However, using short planning period lengths drastically increases the size of the considered model. In the Complex production system, the applied algorithms use a planning period length of 24 hours, although a planning period length of 1 hour would be required to avoid time discretization defects.

Note also that no optimal ex-post solution can be obtained for the Complex production system because of the size of the resulting IP. Nevertheless, we derive results for the continuous relaxation of the IP given by $0 \leq x_{d t} \leq 1$, which provides an upper bound for the maximum attainable profit.

In the numerical tests, the production process is deterministic. However, because some of the applied heuristics in this study suffer from modeling defects because they do not model the available capacity exactly, they might compute infeasible schedules. That is, if capacities are overestimated, according to the schedule, orders must wait within the production system, which is forbidden under Assumption 3.1. Therefore, the order releases generated by these algorithms are converted to a feasible schedule as follows: Orders are released in the planned planning period if sufficient capacity is available to produce them without waiting; otherwise, released orders are processed in an FCFS manner. This can lead to orders finishing after the planning horizon. For a fair comparison, these orders should not generate positive profit because they use capacity from periods after the planning horizon. Therefore, in this numerical study, orders that finish after the planning horizon earn no profit. However, any costs incurred by these orders are still taken into account. 


\subsection{Numerical results}

In this section, we present the results of the numerical study. In particular, we show that the proposed revenue management approach (MS-RM) works well compared to an ex-post optimal solution. Next, we show that aggregating the production system to a single-stage problem does not lead to satisfactory results in production systems with a complex structure. Additionally, the benefit of using online scheduling is described.

The main results can be seen in Figure 2, which shows the mean relative difference (in \%) of the attained marginal profit over all tested instances compared to PostOpt in all tested production systems for all tested algorithms. Additionally, the $95 \%$ confidence intervals are indicated. The following conclusions can be drawn:

- In the tested cases, MS-RM is very close ( $\leq 4.4 \%$ on average) to an ex-post optimal solution in all considered basic production systems. In the Complex production system, the average performance is still good $(8.75 \%)$, despite time discretization defects. This is a quite impressive result, considering that PostOpt has full demand information, while MSRM makes online decisions.

- A simple FCFS approach does not lead to satisfactory results, which suggests that exploiting customer differentiation is essential in the tested cases.

- Applying a one-stage-based heuristic leads to satisfactory results in most of the considered basic production systems. However, the results are notably worse in production systems with a more complex structure, e.g., in those production systems with multiple product types.

- Deciding about order releases online significantly increases the marginal profit compared to fixing order release decisions for an order on its arrival, as seen by MS-RM outperforming SchedArr.

Note that the maximum computation time for solving the IP for the order release decisions is set to 5 minutes, which was sufficient to find a feasible, but not necessarily optimal, solution. Thus, the results for MS-RM could lead to slightly better results if this time limit is relaxed.

In the following, we discuss the absolute performance of MS-RM, the impact of aggregating the production system, and the impact of making online order release decisions in more detail. To this end, we also examine order fill rates (i.e., the ratio of accepted and available orders) for all order types and the resulting holding/backlog costs per order, which are described in Tables 6 and 


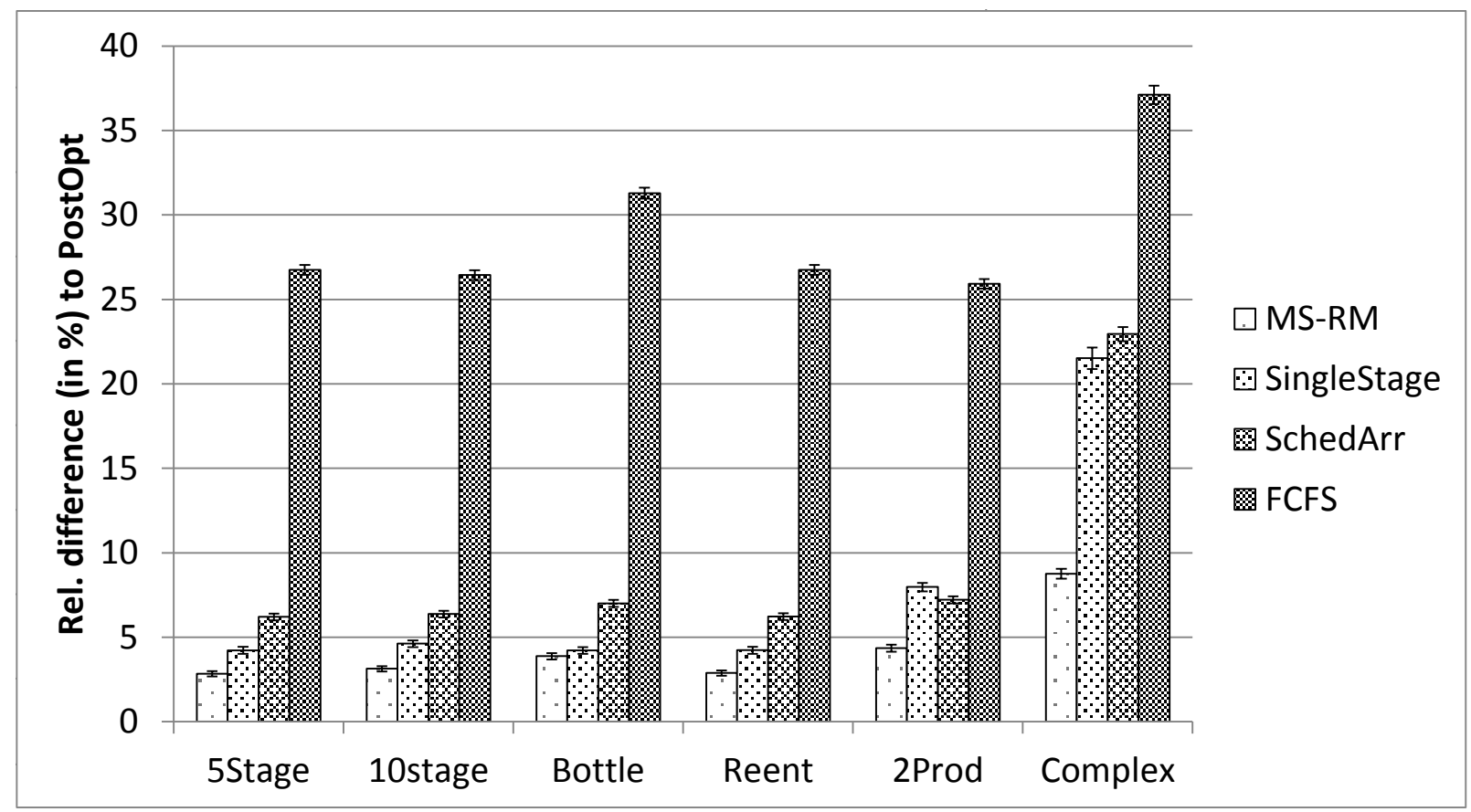

Figure 2: Relative differences in attained marginal profit of heuristics compared to an ex-post optimal solution in various production environments

7, respectively. Structural results for PostOpt in the Complex production system are unavailable because no integer optimal solution can be computed, as discussed above. In Table 7, the costs that were planned by the algorithm are shown in addition to the actual holding/backlog costs because time discretization defects occur in the Complex production system. This is abbreviated as Complex (planned).

\section{Performance of revenue management approach}

In this section, we further investigate the performance of MS-RM compared to an ex-post optimal solution and the FCFS heuristic.

As Figure 2 shows, MS-RM clearly outperforms the FCFS heuristic in all production systems, showing that the considered problem cannot be solved using this simple heuristic. The poor performance might be explained by the fact that FCFS does not differentiate between order types as shown in Table 6, while PostOpt and MS-RM both accept significantly more high-value orders. Additionally, although FCFS does not create backlog costs by definition, the resulting holding costs are rather high, see Table 7.

MS-RM is very close to PostOpt $(\leq 4.4 \%$ on average $)$ in all considered production systems 


\begin{tabular}{rccccc}
\hline & PostOpt & MS-RM & SingleStage & SchedArr & FCFS \\
\hline 5stage & $99.9 / 99.0 / 66.0$ & $98.0 / 95.6 / 68.7$ & $98.1 / 95.9 / 71.3$ & $97.8 / 95.0 / 55.3$ & $71.6 / 71.5 / 73.2$ \\
10stage & $99.5 / 97.5 / 65.4$ & $97.4 / 94.1 / 66.4$ & $97.6 / 96.0 / 71.1$ & $97.8 / 93.1 / 55.6$ & $71.6 / 71.5 / 73.2$ \\
Bottle & $99.8 / 93.9 / 24.0$ & $97.2 / 93.1 / 20.1$ & $97.8 / 94.0 / 19.9$ & $97.3 / 89.3 / 12.6$ & $61.0 / 60.7 / 62.4$ \\
Reent & $99.9 / 99.0 / 66.0$ & $97.9 / 95.6 / 68.8$ & $98.1 / 95.9 / 71.3$ & $97.7 / 95.0 / 55.3$ & $71.6 / 71.5 / 73.2$ \\
2Prod-P1 & $100.0 / 99.3 / 65.5$ & $97.5 / 94.6 / 61.1$ & $99.1 / 97.7 / 70.8$ & $97.7 / 94.6 / 54.4$ & $70.9 / 71.3 / 72.8$ \\
2Prod-P2 & $100.0 / 99.7 / 64.4$ & $98.1 / 96.4 / 63.0$ & $99.0 / 97.8 / 71.0$ & $98.0 / 94.7 / 54.4$ & $74.4 / 74.7 / 74.6$ \\
Compl-P1 & n.a. & $91.1 / 91.4 / 74.4$ & $93.5 / 92.9 / 77.4$ & $83.2 / 83.4 / 55.7$ & $34.7 / 34.8 / 35.6$ \\
Compl-P2 & n.a. & $96.6 / 86.9 / 39.2$ & $96.4 / 95.3 / 62.8$ & $87.1 / 65.6 / 29.8$ & $76.4 / 76.9 / 76.5$ \\
\hline
\end{tabular}

Table 6: Overall averages of order fill rates for high/medium/low-value order types

\begin{tabular}{rcccccc}
\hline & PostOpt & MS-RM & SingleStage & SingleStagePlanned & SchedArr & FCFS \\
\hline 5stage & $2.8 / 0.1$ & $3.8 / 1.1$ & $3.9 / 2.7$ & $4.9 / 1.1$ & $1.6 / 5.3$ & $9.8 / 0.0$ \\
10stage & $2.0 / 0.2$ & $2.7 / 1.4$ & $2.7 / 3.4$ & $4.2 / 1.3$ & $1.1 / 5.7$ & $7.3 / 0.0$ \\
Bottle & $3.0 / 0.4$ & $4.1 / 2.2$ & $4.3 / 2.8$ & $5.2 / 2.0$ & $1.5 / 5.6$ & $10.1 / 0.0$ \\
Reent & $2.8 / 0.1$ & $3.9 / 1.2$ & $3.9 / 2.8$ & $5.0 / 1.1$ & $1.6 / 5.4$ & $10.0 / 0.0$ \\
2Prod & $2.2 / 0.2$ & $2.5 / 2.4$ & $2.0 / 7.5$ & $4.4 / 0.6$ & $1.1 / 6.9$ & $9.8 / 0.0$ \\
Complex & n.a. & $1.3 / 19.0$ & $0.3 / 31.0$ & $2.2 / 5.9$ & $0.4 / 27.9$ & $6.4 / 0.1$ \\
Complex (planned) & n.a. & $4.1 / 8.4$ & $2.2 / 5.9$ & $2.2 / 5.9$ & $1.0 / 21.5$ & $9.0 / 0.0$ \\
\hline
\end{tabular}

Table 7: Overall averages of holding/backlog costs per order

\begin{tabular}{lrcccccc}
\hline & & 5Stage & 10stage & Bottle & Reent & 2Prod & Complex \\
\hline Mean & $100 \%$ & 2.2 & 2.4 & 3.5 & 2.2 & 3.2 & 7.1 \\
& $110 \%$ & 2.9 & 3.2 & 4.1 & 3.0 & 5.0 & 9.1 \\
& $120 \%$ & 3.3 & 3.8 & 4.0 & 3.5 & 4.9 & 10.1 \\
\hline CV & 0.5 & 2.3 & 2.6 & 3.0 & 2.4 & 3.8 & 8.8 \\
& 0.75 & 3.3 & 3.6 & 4.7 & 3.3 & 4.9 & 8.7 \\
\hline \multirow{2}{*}{ ProfitHet } & low & 2.8 & 3.2 & 4.1 & 2.9 & 4.9 & 8.9 \\
& med & 2.9 & 3.1 & 3.9 & 3.0 & 4.5 & 8.6 \\
& high & 2.8 & 3.1 & 3.6 & 2.8 & 3.7 & 8.7 \\
\hline
\end{tabular}

Table 8: Overall averages of performance of MS-RM in different production systems 
except for Complex. The good performance is supported by the other performance indicators. The order fill rates for MS-RM and PostOpt are very close although PostOpt still accepts more orders because it has full knowledge of all incoming demand upfront (cf. Table 6). In addition, it seems that the order differentiation of MS-RM is not strict enough compared to PostOpt. Also, the holding and backlog costs are on a similar level, as shown in Table 7. Again, PostOpt generates lower costs because order release decisions can be performed with the knowledge of all accepted orders.

The performance of MS-RM in the Complex production system is slightly worse than for the other production systems because discretization defects occur in modeling this production system, as described in Section 6.1. Therefore, capacity is overestimated, leading to $4.5 \%$ of the accepted orders finishing after the planning horizon and thus not adding to the earned profit. Additionally, the backlog costs increase from the planned 8.4 per order to an actual 19 per order, as shown in Table 7.

Table 8 shows the impact of the varied parameters on the performance of MS-RM. Here, the relative difference from an ex-post optimal solution is depicted for varying loads of the system, the coefficient of variation of the incoming demand, and the profit heterogeneity (abbreviated as ProfHet) of the orders.

The performance of MS-RM relative to PostOpt decreases with an increasing load. One reason for this is that there are more orders to decide about and, as a result, more mistakes to make.

MS-RM also performs worse for a higher CV in all production system except for Complex. This can be explained by higher fluctuations of demand. Therefore, scenarios in which the computed bid prices are unsuitable for the actual demand arriving occur more frequently. In the Complex production system, this factor seems to play a lesser role. One reason for this might be that because the production system is not modeled exactly, in this case, the impact of the time discretization defects is dominant.

It seems that a low profit heterogeneity leads to a slightly better performance. However, the difference is rather small. On the one hand, the impact of having to reject a high-value order is smaller if the profit heterogeneity is low, but on the other hand, it is easier to differentiate between orders in the case of high profit heterogeneity.

\section{Impact of aggregating the production system}

In this section, we examine the impact of aggregating the production system to a single-stage system. To this end, we compare the SingleStage approach with MS-RM and the ex-post optimal 
solution.

Figure 2 shows that this approach leads to satisfactory results if the production system is simple. However, if, e.g., multiple product types are available, the modeling defects that stem from the aggregation lead to poor results. Table 9 shows the percentage of accepted orders that finish after the planning horizon. These orders do not contribute to overall profit. For the simple production systems, this number is rather low, while for the production systems with two product types, a significant number of accepted orders cannot be produced within the planning horizon. The same result can be observed when comparing the planned and the actual backlog costs in Table 7. While for the simple production systems, the planned and actual realized costs are very similar, they drastically increase in the 2 Prod and Complex production systems.

\begin{tabular}{cccccc}
\hline 5stage & 10stage & Bottle & Reent & 2Prod & Complex \\
\hline 1.0 & 1.6 & 0.5 & 1.0 & 3.5 & 13.1 \\
\hline
\end{tabular}

Table 9: Percentage of the accepted orders finishing after the planning horizon in the SingleStage approach

\section{Impact of using online scheduling}

In this section, the impact of making order release decisions online relative to fixing order release decisions on order arrival is discussed. To this end, we compare SchedArr with MS-RM and PostOpt.

Figure 2 shows that there is a significant benefit to being able to reschedule orders instead of fixing the release dates on arrival. This can be explained by the level of knowledge on the whole set of orders to be scheduled. While PostOpt has full knowledge of all incoming demand and MS-RM at least has the flexibility to change planned order releases if further orders have arrived, SchedArr must determine the order release date for an order immediately on its arrival. In this way, especially in complex production systems, inappropriate order release decisions can lead to resources that can no longer be used. Therefore, SchedArr cannot accept as many orders as MS-RM or PostOpt (cf. Table 6).

Table 10 shows that most of the orders are actually rescheduled in MS-RM. Most of the orders are preponed, which might explain why the backlog costs decrease. However, some orders are also delayed, perhaps allowing more orders to be accepted.

As seen in Table 7, SchedArr generates high backlog costs, which may be related to the chosen order release policy. Order release dates are chosen such that they minimize the sum 


\begin{tabular}{lcccccc}
\hline & 5Stage & 10Stage & Bottle & Reent & 2Prod & Complex \\
\hline Percentage Preponed & 59.5 & 69.9 & 58.9 & 60.2 & 68.3 & 48.8 \\
Percentage Delayed & 13.6 & 15.2 & 11.9 & 12.5 & 14.1 & 18.1 \\
\hline
\end{tabular}

Table 10: Percentage of orders rescheduled in MS-RM

of the bid prices for the used resources and the backlog costs. Bid prices tend to be lower for capacity in late planning periods because, for these periods, less capacity is already planned for fixed order releases. This might be a reason why many orders are backlogged using SchedArr.

\section{Conclusion and further research}

In the present paper, we consider the order acceptance and order release decisions in an orderdriven multi-stage production environment. To the best of our knowledge, this problem has not yet been studied in the literature. We describe this problem as an SDP.

To solve instances of realistic size, we develop a bid-price-based revenue management approach where bid prices are computed using an RLP approach. The bid prices are aggregated to estimate the opportunity costs of using resources for specific orders. The order acceptance and order release decisions are made using an LP, which captures the stochastic nature of the demand via the previously computed opportunity costs.

In a numerical study, we show that the presented approach works well for various production systems compared to an ex-post optimal solution. Even for the Complex production system, which cannot be modeled exactly due to the high computational burden, the performance is only slightly worse. The application of a simple FCFS heuristic leads to unsatisfactory results in all tested production systems, showing that order differentiation is important in the considered settings. Moreover, the benefit of the presented approach compared to existing approaches from the literature, which make simplifying assumptions, is shown.

In the present paper, set-up times are neglected, and processing times are assumed to be deterministic. This is not always the case in real-world production facilities, and it provides a good avenue for further research. Nevertheless, the present paper is a next step in the direction toward enabling the application of revenue management in complex real-world production environments as well. 


\section{A Appendix}

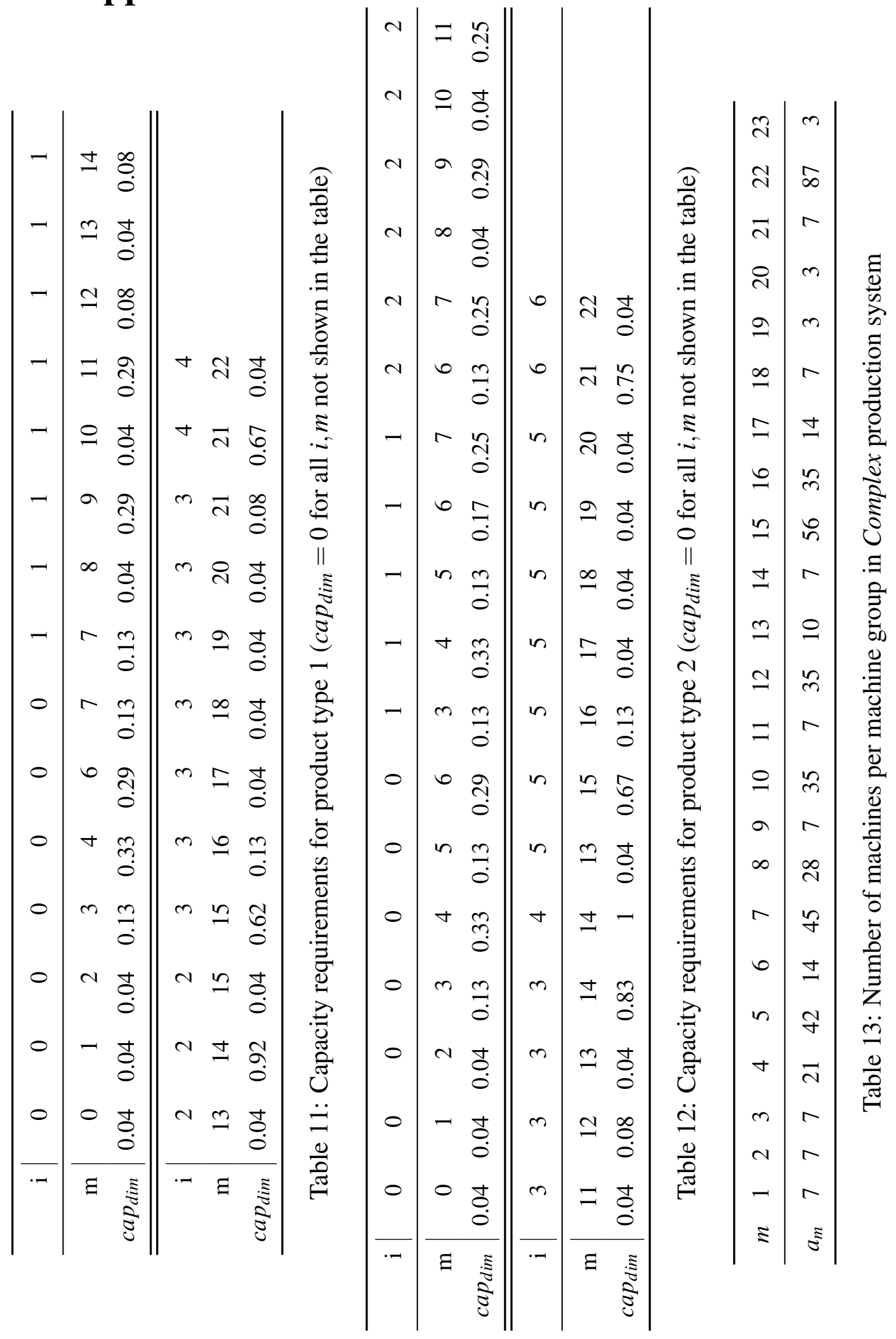




\section{References}

Agrawal, N. and S. Smith (1996). Estimating negative binomial demand for retail inventory management with unobservable lost sales. Naval Research Logistics 43, 839-861.

Barut, M. and V. Sridharan (2005). Revenue Management in Order-Driven Production Systems. Decision Sciences 36(2), 287 - 317.

Brown, A., H. Lee, and R. Petrakian (2000). Xilinx improves its semiconductor supply chain using product and process postponement. Interfaces 10(5), 65-80.

Chevalier, P., A. Lamas, L. Lu, and T. Mlinar (2015). Revenue management for operations with urgent orders. European Journal of Operational Research 240(2), 476-487.

Easton, F. and D. R. Moodie (1999). Pricing and lead time decisions for make-to-order firms with contingent orders. European Journal of Operational Research 116(2), 305-318.

Ehm, H., H. Wenke, L. Monch, T. Ponsignon, and L. Forstner (2011). Towards a supply chain simulation reference model for the semiconductor industry. Proceedings of the 2011 Winter Simulation Conference (WSC), 2119-2130.

Ehrenberg, A. S. C. (1959). The pattern of consumer purchases. Applied Statistics 8(1), 26-41.

Gallego, G., G. Iyengar, R. Phillips, and A. Dubey (2004). Managing flexible products on a network. Technical Report TR-2004-01, Columbia University.

Gallego, G. and R. Phillips (2004). Revenue Management of Flexible Products. Manufacturing \& Service Operations Management 6(4), 321-337.

Gao, L., S. H. Xu, and M. O. Ball (2012). Managing an available-to-promise assembly system with dynamic short-term pseudo-order forecast. Management Science 58(4), 770-790.

Gönsch, J., S. Koch, and C. Steinhardt (2014). Revenue management with flexible products: The value of flexibility and its incorporation into DLP-based approaches. International Journal of Production Economics.

Guhlich, H., M. Fleischmann, and R. Stolletz (2015). Revenue management approach to due date quoting and scheduling in an assemble-to-order production system. OR Spectrum.

Hackman, S. and R. Leachman (1989). A general framework for modeling production. Management Science 35(4), 478-495. 
Hall, N. G. and C. Sriskandarajah (1996). A Survey of Machine Scheduling Problems with Blocking and No-Wait in Process. Operations Research 44(3), 510-525.

Harris, F. d. and J. Pinder (1995). A revenue management approach to demand management and order booking in assemble-to-order manufacturing. Journal of Operations Management 13(4), 299-309.

Hintsches, A., T. S. Spengler, T. Volling, K. Wittek, and G. Priegnitz (2010). Revenue Management in Make-To-Order Manufacturing: Case Study of Capacity Control at ThyssenKrupp VDM. Business Research 3(2), 173-190.

Kuhn, H. and F. Defregger (2005). Revenue management for a make-to-order company with limited inventory capacity. OR Spectrum 29(1), 137-156.

Li, X., J. Wang, and R. Sawhney (2012). Reinforcement learning for joint pricing, lead-time and scheduling decisions in make-to-order systems. European Journal of Operational Research 221(1), 99-109.

Meyr, H. (2009). Customer segmentation, allocation planning and order promising in make-tostock production. OR Spectrum 31(1), 229-256.

Modarres, M. and M. Sharifyazdi (2009). Revenue management approach to stochastic capacity allocation problem. European Journal of Operational Research 192(2), 442-459.

Petrick, A., J. Gönsch, C. Steinhardt, and R. Klein (2010). Dynamic control mechanisms for revenue management with flexible products. Computers \& Operations Research 37(11), 20272039.

Petrick, A., C. Steinhardt, J. Gönsch, and R. Klein (2012). Using flexible products to cope with demand uncertainty in revenue management. OR Spectrum 34(1), 215-242.

Quante, R., M. Fleischmann, and H. Meyr (2009). A Stochastic Dynamic Programming Approach to Revenue Management in a Make-to-Stock Production System. Erasmus Research Institute of Management, Erasmus University.

Simpson, R. W. (1989). Using network flow techniques to find shadow prices for market and seat inventory control. Technical report.

Spengler, T., S. Rehkopf, and T. Volling (2007). Revenue management in make-to-order manufacturing - an application to the iron and steel industry. OR Spectrum 29(1), 157-171. 
Spengler, T. S., T. Volling, and A. Hintsches (2008). Integration von Revenue Management Konzepten in die Auftragsannahme - konkretisiert für Unternehmen der eisen- und stahlerzeugenden Industrie. ZFB Special Issue (4), 125-151.

Stadtler, H. (2008). Production planning and scheduling. In H. Stadtler and C. Kilger (Eds.), Supply Chain Management and Advanced Planning, pp. 199-216. Springer Berlin Heidelberg.

Talluri, K. and G. Van Ryzin (1998). Analysis Network of Controls for Revenue Management. Management Science 44(11), 1577-1593.

Talluri, K. and G. Van Ryzin (1999). A randomized linear programming method for computing network bid prices. Transportation Science, 207-216.

Talluri, K. and G. Van Ryzin (2004). The theory and practice of revenue management. Kluwer Academic Publishers.

Tang, L., J. Liu, A. Rong, and Z. Yang (2001). A review of planning and scheduling systems and methods for integrated steel production. European Journal of Operational Research 133(1), $1-20$.

Volling, T., D. Eren Akyol, K. Wittek, and T. S. Spengler (2012). A two-stage bid-price control for make-to-order revenue management. Computers \& Operations Research 39(5), 10211032.

Watanapa, B. and A. Techanitisawad (2005). Simultaneous price and due date settings for multiple customer classes. European Journal of Operational Research 166(2), 351-368.

Williamson, E. L. (1992). Airline network seat inventory control: Methodologies and revenue impacts. Ph. D. thesis, MIT, Cambridge.

Wismer, D. a. (1972). Solution of the Flowshop-Scheduling Problem with No Intermediate Queues. Operations Research 20,689-697. 